IOS Press

\title{
Editorial
}

\section{An outstanding journal for transformational transdisciplinary research}

Integrated Computer-Aided Engineering (ICAE) is a forum of outstanding quality that has the reputation of being a top journal in Computer Science, Artificial Intelligence, Engineering, and Interdisciplinary Applications. For nearly three decades, ICAE has been advancing innovative multidisciplinary research with absolute consistency, and maintaining the highest standards of quality without compromise. The scientific community has honored ICAE with its trust and a very high impact factor.

ICAE achieved this extraordinary status thanks to its indefatigable, far-sighted, and ingenious founding Editor-in-Chief, Professor Hojjat Adeli and his meticulous and rigorous editorial work on each submission. First, the prospective authors are requested to submit an overview on the techniques in the same field of the submitted manuscript to verify their awareness of the latest developments and leaders in the field. Authors will be asked to indicate that the submission is ready to be considered by the journal by checking off all the items regarding ethical requirements for the authors such as originality and exclusiveness of the submission, acknowledgment of authorship and sources, and absence of plagiarism and conflict of interest. The journal requires each author to submit a Similarity Report using a similarity checking or plagiarism software. The similarity to any other archival source is required to be less than $1 \%$.

Then, in the reviewing process, each article goes through multiple rounds of review by at least five reviewers, sometimes as many as 10 , to ensure the paperąŕs high quality. Prof. Adeliąís personalized and caring manuscript submission and review system, as opposed to increasingly ubiquitous impersonal automated electronic manuscript processing systems and the superficial bean counting by many journal editors makes ICAE stand out and be special. The comprehensive peer review of this 5-review journal is able not only to act as a filter to determine the validity, significance and originality of the work to ensure only high-quality research is published but also to improve the quality of research submitted for publication through constructive suggestions made by a large number of reviewers. In return, journal contributors commit to provide detailed reviews, as a Conscientious Reviewer, for papers submitted to the journal as the journal reviewers are often chosen among contributors to the journal. This is how thoughtful and detailed reviews are obtained in a timely fashion. The journal contributors and Conscientious Reviewers collectively make the Society of ICAE.

To sum up, the uniqueness and qualities of ICAE include its transdisciplinary nature, the very high selection criteria, short review time and production cycle, and a most-dedicated and remarkable Editor-in-Chief who is the author of many pioneering and transdisciplinary research articles and high-technology books. It is certain that ICAE will continue to be a leading forum for cross-disciplinary research in the domains such as Artificial Intelligence, Computer Science, and Engineering. Happy 30th to ICAE, and let us continue this tradition of excellence into the next decade.

\section{Yuping Wang}

Professor

School of Computer Science and Technology,

Xidian University, Xi'an, Shaanxi, China

E-mail: ywang@xidian.edu.cn 